\title{
AVALIAÇÃO DO EQUILÍBRIO, FORÇA E VELOCIDADE DA MARCHA DE IDOSAS ATIVAS
}

Ana Caroline Rippi Moreno, Elaine Aparecida Lozano da Silva, Lucas Oliveira Klebis, José Henrique Piedade Cardoso, Claudia Regina Sgobbi de Faria, Regina Celi Trindade Camargo.

Universidade Estadual Paulista - UNESP, Curso de Fisioterapia, Presidente Prudente, SP.

\section{RESUMO}

Relacionado ao envelhecimento está o risco de quedas, causadas por alterações no equilíbrio, marcha e força muscular. Um questionário atual que avalia de forma completa todos esses itens é o Short Physical Performance Battery (SPPB). Este artigo tem como objetivo, analisar as três etapas do teste SPPB, em relação a capacidade física de idosas ativas. Foi avaliado escolaridade, estado civil, IMC e teste SPPB em 50 idosas ativas. A análise estatistica utilizada foi ANOVA, com significância $p<0,05$. A média de idade foi de $70,7 \pm 6,9$, IMC de $28,4 \pm 4,8$, maior escolaridade entre 2 20 e 3 o grau e $64 \%$ eram casadas. No teste SPPB, $90 \%$ apresentaram resultado Bom, dessas $50 \%$ conseguiram pontuação máxima. O maior déficit foi na força muscular, com diferença significante quando comparado com velocidade e equilíbrio. Pode-se concluir que o programa de atividade física regular desenvolve auxilio nos riscos de quedas, obtendo como maior déficit a força de membros inferiores.

Palavras chave: Atividade física, idosas e equilíbrio.

\section{BALANCE EVALUATION, STRENGTH AND OLD RUNNING SPEED LIVE UNATI PROGRAM PARTICIPANTS}

\begin{abstract}
Related to aging is the risk of falls caused by changes in the balance, gait and muscle strength. A current questionnaire that evaluates thoroughly all of these items is the Short Physical Performance Battery (SPPB). This article aims to analyze the three stages of SPPB test in relation to physical capacity of active elderly women. education was evaluated, marital status, BMI and SPPB test 50 active women. The statistical analysis used was ANOVA, with significance $p<0.05$. The average age was $70.7 \pm 6.9$, BMI $28.4 \pm 4.8$, higher education between 2 nd and 3rd degree and $64 \%$ were married. In SPPB test, $90 \%$ had results Well, these $50 \%$ were able to score maximum. The largest deficit was in muscle strength, with significant difference when compared with speed and balance. It can be concluded that regular physical activity program develops aid in the risk of falls, getting as largest deficit the strength of the lower limbs.
\end{abstract}

Keywords: physical activity, elderly and balance. 


\section{INTRODUÇÃO}

Estima-se que no Brasil a população idosa ocupe aproximadamente $13 \%$ de sua população total, dado esse referente ao aumento significativo da expectativa de vida em relação a décadas passadas (1). Acredita-se que o número de idosos no Brasil deverá evoluir muito mais rápido do que a média internacional, estimando que a quantidade de idosos vá duplicar no mundo, porém triplicar no Brasil, atingindo em 2050 a porcentagem de 30\% em relação à população total (2).

O processo de envelhecimento acarreta em alterações metabólicas e fisiológicas inevitáveis no corpo humano, desenvolvendo diversas patologias crônicas e prejuízos na capacidade funcional do idoso, bem como na qualidade de vida (3). Desse modo, a queda na qualidade de vida do idoso, desenvolve um declínio físico e mental, com diversas alterações, tais como a redução do equilíbrio, força e flexibilidade muscular e a propensão de desenvolver fragilidade. Essas alterações predispõem o idoso ao risco de quedas além de dificultar a realização das atividades de vida diárias (AVD) (4).

Quedas podem ser definidas como episódios de desequilíbrio que levam o indivíduo ao chão, e ocorrem com mais frequência em indivíduos com idade igual ou superior a 60 anos (4). Além disso, a queda é resultante da interação entre vários fatores, podendo ser intrínsecos e extrínsecos (5). Os fatores intrínsecos podem ser definidos como aqueles relacionados ao próprio sujeito, o qual pode apresentar redução da função dos sistemas que compõem o controle postural, doenças, transtornos cognitivos e comportamentais. Como fatores extrínsecos têm-se aqueles relacionados ao ambiente, tais como iluminação, superfície para deambulação, tapetes soltos, degraus altos ou estreitos (5).

Dentre os fatores preponderantes pelas quedas na população idosa, encontram-se as alterações do equilíbrio corporal, que refletem um processo dependente da integração do sistema vestibular, visual, proprioceptivo, reações neuromusculares, de força muscular e tempo de reação (6). Sendo assim torna-se de suma importância a integração do equilíbrio, marcha e velocidade, a fim de prevenir agravos na saúde do idoso, tal como as quedas.

Dentre os testes com objetivo de analisar possíveis alterações no equilíbrio, força muscular e velocidade do idoso encontra-se o teste Short Physical Performance Battery (SPPB)(7). Esse teste combina três componentes: equilíbrio, velocidade de caminhada e força muscular (8), que quando avaliados e interpretados de forma associada, são capazes de verificar possíveis riscos de quedas, alterações funcionais e interferir diretamente na qualidade de vida (9).

O exercício físico regular vem sendo citado em artigos científicos como uma das principais ferramentas preventivas utilizadas na população idosa (10-13). A atividade física regular pode evitar, tardar ou até mesmo melhorar quadros presentes no envelhecimento, sugerindo que idosos ativos sejam capazes de realizar suas AVD sem dificuldades (14), além de reduzir possíveis riscos como as quedas $(14,15)$.

O objetivo desse estudo foi analisar e comparar as três etapas do teste SPPB, em relação à capacidade física de uma população idosa ativa.

\section{METODOLOGIA}

Após a aprovação do comitê de ética em pesquisa, da cidade de Presidente Prudente (CAEE n 56496816.3.0000.5402) foi realizado esse estudo transversal com 50 idosas ativas, integrantes do programa de fisioterapia preventiva para a terceira idade, da Universidade aberta da Terceira Idade (UNATI), realizado no Centro de Atendimentos em Fisioterapia e Reabilitação (CEAFIR) e Núcleo Morumbi, da FCT/UNESP - campus de Presidente Prudente.

As voluntárias foram devidamente informadas sobre os procedimentos e objetivos do presente estudo e após concordarem, assinaram um termo de consentimento livre e esclarecido.

Todas as participantes do presente estudo eram do sexo feminino com idade igual ou superior 60 anos, eram participantes de um dos programas de fisioterapia preventiva para a 
terceira idade citados acima e possuíam no mínimo 75\% de frequência. Foi considerado critérios de exclusão somente participantes com alterações cognitivas detectadas pelo Mini Exame do Estado Mental (MEEM) (16), segundo escore de Brucki et al.(2003) (17).

\section{COLETA DE DADOS}

Caracterização: O estudo contou com coleta dados pessoais, sendo: idade, estado civil (considerando-se: solteiro, casado, divorciado e viúvo) e escolaridade (analfabeta, primeiro grau completo, segundo grau completo e terceiro grau completo), avaliação antropométrica (peso e altura para cálculo do IMC), seguindo a literatura (18), utilizando balança antropométrica digital da marca Líder e aplicação do teste Short Physical Performance Battery (SPPB).

Questionários: Os questionários MEEM e SPPB, foram aplicados por fisioterapeutas, especialistas e residentes no setor de Gerontologia na UNESP de Presidente Prudente. As coletas foram realizadas em julho de 2015.

SPPB: Estudos apontam o teste SPPB como um instrumento prático e eficaz para a avaliação do desempenho físico e o rastreamento de idosos com riscos futuros (9). O instrumento possui três subdivisões, sendo equilíbrio em três posições diversificadas por 10 segundos, velocidade da marcha em passo habitual por quatro metros e força de membros inferiores com o exercício de sentar e levantar de uma cadeira, cinco vezes em sua velocidade máxima. De forma geral, o objetivo padronizado do teste é avaliar o equilíbrio de forma completa.

O escore total do SPPB é alcançado pela soma das três subdivisões (7), podendo cada uma pontuar 4 pontos, desse modo o valor pode variar de 0 (pior desempenho) a 12 (melhor desempenho), recebendo a seguinte graduação: 0 a 3 pontos: Incapacidade / Desempenho muito ruim; 4 a 6 pontos: Baixo desempenho; 7 a 9 pontos: Moderado desempenho; 10 a 12 pontos: Bom desempenho.

ANÁLISE ESTATíSTICA: Os resultados foram expressos como média \pm desvio padrão. As avaliações estatísticas dos resultados foram feitas pelo programa PRISMA. Para a análise estatística comparativa foi utilizado One Way/ANOVA. As diferenças entre os grupos foram consideradas significantes quando $p<0,05$. Os resultados foram expressos em formas de tabelas e gráficos de colunas.

\section{RESULTADOS}

Foram avaliadas 50 pessoas do sexo feminino, com idade média de $70,7 \pm 6,9$ anos e IMC médio de $28,4 \pm 4,8 \mathrm{~kg} / \mathrm{m}^{2}$.

As participantes apresentaram no Short Physical Performance Battery (SPPB), valor médio de $11,1 \pm 1,1$ pontos. Vale ressaltar que o resultado máximo para o teste é de 12 pontos, sendo avaliado como bom desempenho uma nota superior igual a 10 pontos (Tabela 1 ).

Tabela 01. Caracterização das participantes da pesquisa.

\begin{tabular}{lc}
\hline $\mathbf{N}(\mathbf{5 0})$ & Media \pm DP \\
Idade (anos) & $70,7 \pm 6,9$ \\
Peso $(\mathrm{kg})$ & $67,3 \pm 12,9$ \\
Altura $(\mathrm{m})$ & $1,53 \pm 0,05$ \\
IMC $\left(\mathrm{kg} / \mathrm{m}^{2}\right)$ & $28,4 \pm 4,8$ \\
SPPB & $11,1 \pm 1,1$ \\
\hline
\end{tabular}


Em relação ao grau de escolaridade, em valores porcentuais o grupo analfabeto correspondeu a $5 \%$ das participantes, 1 o grau completo $15 \%$ e $40 \%$ referente ao $2 \circ$ e $3^{\circ}$ grau completos (Figura 1).

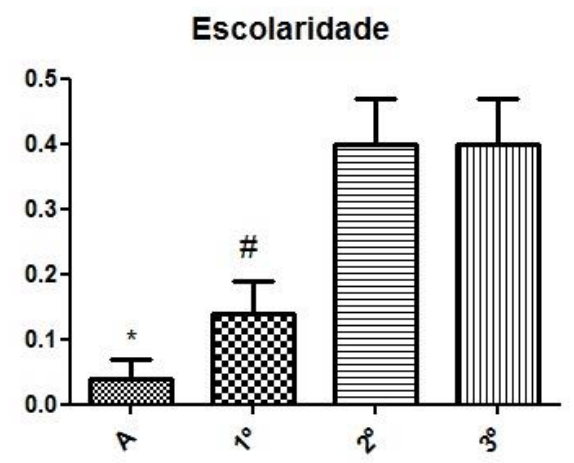

Figura 01. Escolaridade das participantes.Diferença significante $p<0,05$ em: * vs $2 \circ$ e 3ㅇ \# vs $2 \circ$ e 3ㅇ. Considera-se $A=$ Analfabetos, $10=10$ grau completo, $20=20$ grau completo e $30=30$ grau completo.

Quando avaliado o estado civil, encontrou-se um predomínio de mulheres casadas (64\%). Em valores porcentuais, o grupo solteira obteve $6 \%$ das participantes, casada $64 \%$, viúva $28 \%$ e divorciada 2\% (Figura 2).

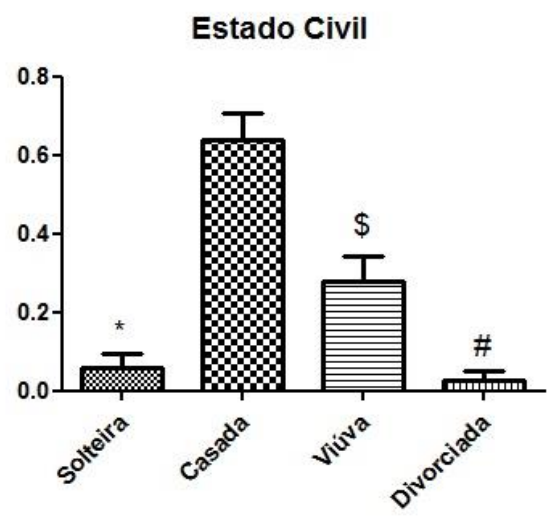

Figura 02. Estado civil das participantes. Diferença significante $p<0,05$ em: * vs casada e viúva; \# vs casada e viúva; \$ vs casada.

Pela aplicação do Short Physical Performance Battery (SPPB), a maioria das participantes apresentaram bom desempenho (90\%), dessas, 50\% atingiram pontuação máxima no teste. Foi considerando como Bom, uma pontuação acima de 10, Moderado entre 7 e 9 pontos, Baixo entre 6 e 4 pontos e Ruim quando abaixo de 3 pontos. O grupo moderado obteve apenas $10 \%$ das participantes e o grupo Baixo e Ruim porcentagem nula (Figura 3). 


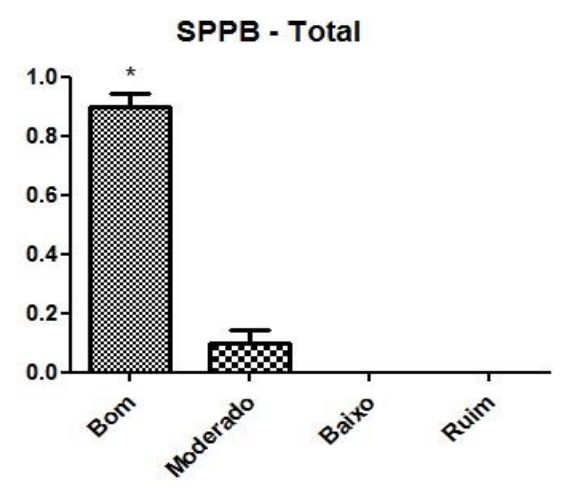

Figura 03. Escore total SPPB. Diferença significante $p<0,05 \mathrm{em}$ : * vs Moderado, Baixo e Ruim.

O questionário SPPB avalia três dimensões, ou seja; equilíbrio, velocidade e força, podendo atingir no máximo 4 pontos em cada dimensão. O principal objetivo dessa análise é verificar o maior déficit das participantes, ou seja, qual dimensão apresenta a menor pontuação.

Segundo o resultado expresso na figura 4, observa-se que a maior dificuldade foi em força muscular (teste sentar e levantar), com diferença significante vs equilíbrio (posição tandem, semi tandem e pés paralelos) e velocidade (caminhada de 4 metros).

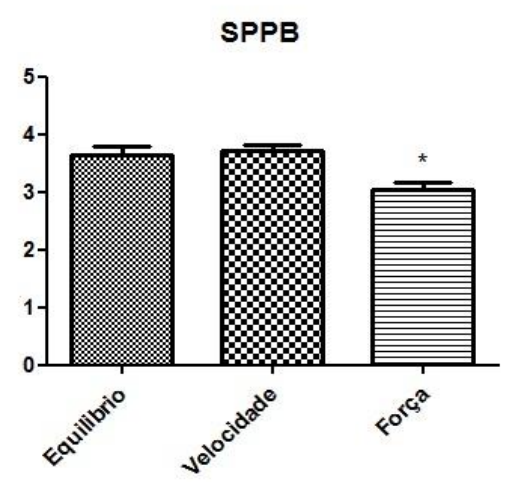

Figura 04. Análise de maior déficit em idosos ativos segundo o questionário SPPB.

Diferença significante $p<0,05$ em: * vs Equilíbrio e Velocidade.

\section{DISCUSSÃO}

Acredita-se que um envelhecimento bem-sucedido vem acompanhado de bem-estar físico e mental, estando associado à independência nas atividades de vida diária, sendo que esses objetivos são alcançados quando acompanhados da pratica regular de atividade física (19).

Segundo o resultado expresso na Figura 3, observa-se que 90\% das participantes atingiram um escore Bom, destas $50 \%$ conseguiram pontuação máxima, além do que, nenhuma se enquadrou no escore baixo ou ruim. Apesar do presente estudo não obter grupo sedentário para uma possível comparação, na literatura, diversas referências apresentam resultados semelhantes ao grupo ativo. Silva (2010), comparou um grupo sedentário com um grupo ativo e seu resultado foi favorável ao grupo ativo em relação ao questionário SPPB, visto que na população sedentária somente $40 \%$ dos participantes atingiram o escore de Bom desempenho, enquanto nos ativos foi de $73 \%(20)$.

Um segundo estudo com resultados positivos ao grupo ativo, também utilizando o SPPB, comprovou que os exercícios resistidos e de capacidade aeróbica, melhoram a capacidade funcional dos idosos, reduzindo o risco de quedas (21). Tais exercícios são semelhantes aos desenvolvidos pela UNATI de Presidente Prudente. 
Sabe-se que idosos que apresentam redução de força muscular de MMII, alterações no equilíbrio e marcha, estão mais propícios ao risco de quedas (22). Ao analisar a figura 4, observase que a maior dificuldade das participantes está na força muscular de MMII. A força muscular e a contração diminuem com o envelhecimento (23). Portanto o envelhecimento é acompanhado de redução da massa muscular, força, atrofia e redução das fibras (24).

O declínio de força muscular no MMII do idoso, também classificado como sarcopenia, compromete diretamente a capacidade funcional, podendo futuramente comprometer também os outros tópicos avaliados pelo SPPB, como velocidade da marcha e equilíbrio, relacionados com as atividades de vida diária, por exemplo, caminhar, subir escadas, levantar-se da cama, vestir-se, banhar-se, entre outros mencionados na literatura $(25,26,27,28)$.

No trabalho de Alves (29), foi realizada hidroginástica em idosos durante três meses, e após reavaliados foi observado um ganho de força, velocidade e equilíbrio, obtendo como resultado, maior dificuldade no idoso quanto à força de MMII, no entanto essa redução foi ainda maior no grupo sedentário, sendo que $46 \%$ da população ativa atingiu nota máxima e $46 \%$ da população sedentária, mínima. Assim sendo, pode-se entender que a pratica regular de atividade física é ideal e fundamental para o idoso, envolvendo os três aspectos analisados no questionário SPPB, força, velocidade e equilíbrio $(30,31)$, além do que, as alterações do envelhecimento são condições potencialmente reversíveis acompanhadas de um bom desempenho físico, buscando programas preventivos, com profissionais fisioterapeutas e educadores físicos $(21,27,28)$.

\section{CONCLUSÃO}

Conclui-se que são as idosas com maior grau de instrução e casadas, que procuram a pratica regular de atividade física oferecida pela UNATI, que a maioria se encontra com baixo risco de quedas de acordo com o questionário SPPB, e que a principal dificuldade é a redução de força muscular de MMII.

\section{REFERÊNCIAS}

Jornal de Brasília. Idosos representam 13\% da população brasileira. [Internet]. 2015 [acessado em 2016 maio 15. Disponível em: http://jornaldebrasilia.com.br/noticias/brasil/608656/idososrepresentam-13-da-populacao-brasileira/

Sordi J. Zh Vida. Número de idosos quase triplicará no Brasil até 2050, afirma OMS. [Internet]. 2015 [acessado em 2016 maio 15]. Disponível em: http://zh.clicrbs.com.br/rs/vida-eestilo/vida/noticia/2015/09/numero-de-idosos-quase-triplicara-no-brasil-ate-2050-afirma-oms4859566.html

Fechine B.R.A, Trombieri N.O processo de envelhecimento: As principais alterações que acontecem com o idoso com o passar dos anos. Interscienceplace. 2012 mar: 20 (1): 107.

Lima MSD. Relação da força, da flexibilidade e do nível de atividade física no equilíbrio motor de idosos. [Trabalho de conclusão de curso]. Curitiba: Universidade Tecnológica Federal do Paraná, 2011.

Almeida ST, Soldera CLC, Carli GA, et all. Análise de fatores extrínsecos e intrínsecos que predispõem a quedas em idosos. Rev Assoc Med Bras 2012; 58(4):427-433.

https://doi.org/10.1590/S0104-42302012000400012.

Fiedler MM, Peres KG. Functional status and associated factors among the elderly in a southern Brazilian city: a population-based study. Cad Saúde Pública 2008;24(2):409-15. 
Guralnik JM, Ferrucci L, Simonsick EM, Salive ME, Wallace RB. Lower extremity function in persons over the age of 70 years as predictor of subsequent disability. The New England Journal of Medicine. 1995 Mar;232(9):556-61. https://doi.org/10.1056/NEJM199503023320902.

Nakano MM. Versão Brasileira da Short Physical Performance Battery - SPPB: Adaptação Cultural e Estudo da Confiabilidade. Campinas. Dissertação [Mestrado] - Faculdade de Educação, Universidade Estadual de Campinas; 2007.

Marchon RM, Cordeiro RC, Nakano MM. Capacidade funcional: estudo prospectivo em idosos residentes em uma instituição de longa permanência. As. Bras. Geriatr. Gerontol. 2010;13(2):20314. https://doi.org/10.1590/S1809-98232010000200005.

Cheik NC, Reis IT, Heredia RAG, et all. Efeito do exercício físico e da atividade física na depressão e ansiedade em indivíduos idosos. Revista Ciência e Movimento. Brasília, 2003; 11(3):45-52.

Souza WC, Mascarenhas LPG, et all. Exercício Físico na promoção da saúde na terceira idade. Revista Saúde Meio Ambiente. Contestado, 2015;4(1):55-65.

Goersch CM, Lomboglia F, Costa RG, et all. Efeito do tempo de prática de exercício físico na aptidão fisica relacionada a saúde de mulheres idosas. Revista Brasil Promoção e Saúde. Fortaleza, 2014;27(1):29-36.

Carvalho MJ, Fernandes R, Mota J. Efeito do exercício físico na aptidão física de mulheres idosas. Revista Kinesis. Santa Maria, 2001;24:197-206.

Rebelatto JR, Calvo JI, Orejuela JR, Portillo JC. Influência de um programa de atividade física de longa duração sobre a força muscular manual e a flexibilidade corporal de mulheres idosas. Rev. Bras. Fisioter. 2006; 10(1): 127-132. https://doi.org/10.1590/S1413-35552006000100017.

Mazo GZ, Liposcki DB, Ananda C, Prevê D. Condições de saúde, incidência de quedas e nível de atividade física dos idosos. Rev. Bras. Fisioter. São Carlos, 2007;11(6): 437-442.

Folstein M F, Folstein S E \& McHugh PR. "Mini-Mental State": a practical method for grading the cognitive state of patients for the clinician. J. Psychiat. Res. 1975: 12:189-98.

https://doi.org/10.1016/0022-3956(75)90026-6.

Brucki SMD et al. Sugestões para o uso do Mini-Exame do Estado Mental no Brasil. Arquivos de Neuro-Psiquiatria, 2003, 61(3):777-781 B. https://doi.org/10.1590/S0004-282X2003000500014.

Junior I.F.F. Padronização de técnicas antropométricas. 1. ed. São Paulo: Cultura Acadêmica: Universidade Estadual Paulista, Pró- Reitoria de Graduação, 2009. p.70.

Henwood TR, Taaffe DR. Improved physical performance in older adults undertaking a short-term programme of high-velocity resistance training. Gerontology 2005;51(2):108-15. https://doi.org/10.1159/000082195.

Silva TO, Freitas RS, Monteiro MR, et al. Avaliação da capacidade física e quedas em idosos ativos e sedentários da comunidade. Rev Bras Clin Med. São Paulo, 2010;8(5):392-8. 
Macedo C, Gazzola JM, Najas M. Síndrome da fragilidade no idoso: importância da fisioterapia. Arq Bras Cienc Saúde 2008;33(3):177-84. https://doi.org/10.7322/abcs.v33i3.154.

Silva A, Almeida GJM. Cassilhas RC, et al. Equilíbrio, coordenação e agilidade de idosos submetidos à prática de exercícios físicos resistidos. Rev Bras Med Esporte 2008;14(2):88-93. https://doi.org/10.1590/S1517-86922008000200001.

Janssen I. Influence of sarcopenia on the development of physical disability: the Cardiovascular Health Study. J Am Geriatr Soc 2006;54(1):56-62. https://doi.org/10.1111/i.15325415.2005.00540.x.

Doherty TJ. Invited review: Aging and sarcopenia. J App Physiol 2003;95(4):1717-27. https://doi.org/10.1152/japplphysiol.00347.2003.

Earles DR, Judge JO, Gunnarsson OT. Velocity training induces power-specific adaptations in highly functioning older adults. Arch Phys Med Rehabil 2001;82(7):872-8.

https://doi.org/10.1053/apmr.2001.23838.

Onder G, Penninx BW, Ferrucci L, et al. Measures of physical performance and risk for progressive and catastrophic disability: results from the Women's Health and Aging Study. J Gerontol A Biol Sci Med Sci 2005;60(1):74-9. https://doi.org/10.1093/gerona/60.1.74.

Davini R, Nunes CV. Alterações no sistema neuromuscular decorrentes do envelhecimento e o papel do exercício físico na manutenção da força muscular em indivíduos idosos. Rev Bras Fisioter 2003;7(3):201-7.

Alves RV, Mota J, Alves MC, et al. Aptidão física relacionada à saúde de idosos: influência da hidroginástica. Rev Bras Med Esporte, 2004;10(1):31-7. https://doi.org/10.1590/S1517$\underline{86922004000100003 .}$

Vreede PL, Samson MM, van Meeteren NL, et al. Functionaltask exercise versus resistance strength exercise to improve daily function in older women: a randomized, controlled trial. J Am Geriatr Soc 2005;53(1)2-10. https://doi.org/10.1111/j.1532-5415.2005.53003.x.

Ferrucci L, Guralnik JM, Salive ME, et al. Effect of age and severity of disability on short-term variation in walking speed: the Women's Health and Aging Study. J Clin Epidemiol 1996;49(10):1089-96. https://doi.org/10.1016/0895-4356(96)00231-4.

Spirduso WW. Dimensões físicas do envelhecimento, São Paulo: Manole; 2005. p. 38-56. 